\title{
Approximate Controllability for Time-Dependent Impulsive Neutral Stochastic Partial Differential Equations with Fractional Brownian Motion and Memory
}

\author{
K. Ramkumar ${ }^{1}$, K. Ravikumar ${ }^{1}$, E. M. Elsayed ${ }^{2 *}$ and A. Anguraj $^{1}$ \\ ${ }^{1}$ Department of Mathematics, PSG College of Arts and Science, Coimbatore, 641 014, India. \\ ${ }^{2}$ Department of Mathematics, Faculty of Science, King Abdulaziz University, Jeddah 21589, Saudi Arabia. \\ ${ }^{*}$ Corresponding author
}

\author{
Article Info \\ Keywords: Approximate controllability, \\ Impulsive systems, Fractional Brownian \\ motion, Delay differential equations. \\ 2010 AMS: 34K50, 93B05, 34A37, \\ 93E03, $60 \mathrm{H} 20$. \\ Received: 7 July 2019 \\ Accepted: 13 July 2020 \\ Available online: 29 September 2020
}

\begin{abstract}
In this manuscript, we investigate the approximate controllability for time-dependent impulsive neutral stochastic partial differential equations with fractional Brownian motion and memory in Hilbert space. By using semigroup theory, stochastic analysis techniques and fixed point approach, we derive a new set of sufficient conditions for the approximate controllability of nonlinear stochastic system under the assumption that the corresponding linear system is approximately controllable. Finally, an example is provided to illustrate our results.
\end{abstract}

\section{Introduction}

Approximate controllability is one of the important fundamental concepts in mathematical control theory and plays an important role in both deterministic and stochastic control systems. Controllability generally means that it is possible to steer a dynamical control system from an arbitrary initial state to an arbitrary final state using the set of admissible controls. The controllability of nonlinear stochastic systems in infinite dimensional spaces has recently received a lot of attentions see $[1-5,10,11,13]$ and the references therein. Moreover, the approximate controllability means that the system can be steered to arbitrary small neighborhood of final state. Further, approximate controllable systems are more prevalent and very often approximate controllability is completely adequate in applications see $[7,8]$ and the references cited therein.

Likewise the theory of impulsive differential equations plays a major role in investigation of qualitative theory. Impulsive differential equations are differential equations involving impulse effect, appear as a natural description of observed evolution phenomena of several real life problems, for detail refer [6]. In other way, many dynamical systems (Physical, Social, Biological, Engineering etc.) can be conveniently expressed in the form of differential equations. In case of physical systems such as air crafts, some external forces act which are not continuous with respect to time and the duration of their effect is near negligible as compared with total duration of original process. Same phenomena's are observed in case of biological systems (e.g. heart beat, blood-flow, pulse frequency), social systems (e.g. price-index frequency, demand and supply of goods) and in many other dynamical systems also such effects are called impulsive effects.

In recent years, stochastic differential equations driven by fractional Brownian motion have attracted much attention due to its a wide applications in a verity of physical phenomena, such as in economic and finance, biology and communication networks. The fractional Brownian motion was introduced by Kolmogorov in 1940 in [9], and later studied by Mandelbrot and Van Ness, who in 1968 provided in [10] a stochastic integral representation of this process in terms of a standard Brownian motion. There has been some recent interest in studying evolution equations driven by fractional Brownian motion. Recently, Lakhel [11] studied controllability results of neutral stochastic delay partial functional integrodifferential equations perturbed by fractional Brownian motion by using the theory of semigroup. Very recently, many interesting works have been done on stochastic differential equations driven by fractional Brownian motion see $[3,11,12,15]$ and the references cited therein.

Email addresses and ORCID numbers: ramkumarkpsg@gmail.com, https://orcid.org/0000-0002-3624-5363 (K. Ramkumar), ravikumarkpsg@gmail.com, https://orcid.org/0000-0002-5635-8517 (K. Ravikumar), emmelsayed@yahoo.com, (E. M. Elsayed), angurajpsg@ yahoo.com, https://orcid.org/0000-0003-0517-2875 (A. Anguraj) 
However, the study of the approximate controllability for time-dependent impulsive neutral stochastic partial differential equations with fractional Brownian motion and memory has not been discussed in the standard literature. Motivated by the above consideration, the goal of this paper is to study the approximate controllability for time-dependent impulsive neutral stochastic partial differential equations with fractional Brownian motion and memory:

$$
\begin{aligned}
d[x(t)-g(t, x(t-r))] & =A(t)[x(t)-g(t, x(t-r))] d t+[f(t, x(t), x(t-r))+B u(t)] d t+\sigma(t) d \mathrm{~B}^{\mathrm{H}}(t), \quad t \neq t_{k}, \quad t \in J:=[0, T], \\
\Delta x\left(t_{k}\right) & =I_{k}\left(x\left(t_{k}^{-}\right)\right), \quad k \in\{1,2, \ldots, m\}, \\
x(t) & =\varphi(t) \in \mathscr{C}_{r}=\mathscr{C}_{\mathfrak{I}_{0}}^{b}([-r, 0] ; \mathrm{H}), \quad-r \leq t \leq 0, \quad r>0 .
\end{aligned}
$$

where $x(\cdot)$ is a stochastic process taking values in a real separable Hilbert space $\mathrm{H} ; A(t): \mathscr{D} \subset \mathrm{H} \rightarrow \mathrm{H}$ is a family of unbounded operators defined on a common domain $\mathscr{D}$, which is dense in the space $\mathrm{H}$ and generates a strong evolution operator $\mathscr{U}(s, t), 0 \leq t \leq s \leq T$ and $g, f: J \times \mathrm{H} \rightarrow \mathrm{H}, \sigma: J \rightarrow \mathscr{L}_{2}^{0}$ are Borel measurable functions and $I_{k}: \mathrm{H} \rightarrow \mathrm{H}, k=1,2, \ldots, m$ are continuous functions. The control function $u(\cdot)$ takes values in $\mathscr{L}_{2}^{\mathfrak{I}}(J, \mathrm{U})$ of admissible control functions for a separable Hilbert space $\mathrm{U}$ and $B$ is a bounded linear operator from $\mathrm{U}$ into $\mathrm{H}$. Furthermore, let $0=t_{0}<t_{1}<\cdots t_{m}<t_{m+1}=T$ be prefixed points, and $\Delta x\left(t_{k}\right)=x\left(t_{k}^{+}\right)-x\left(t_{k}^{-}\right)$, represents the jump of the function $x$ at time with $I_{k}$ determining the size of the jump, where $x\left(t_{k}^{+}\right)$and $x\left(t_{k}^{-}\right)$represent the right and left limits of $x(t)$ at $t=t_{k}$, respectively. Let $\varphi(t):[-r, 0] \rightarrow \mathrm{H}$ is an $\mathfrak{I}_{0}$-measurable random variables independent of the Wiener process $W$ with $\mathbf{E}\left[\sup _{-r \leq s \leq 0}\|\varphi\|_{\mathrm{H}}^{2}\right]<\infty$.

\section{Preliminaries}

Let $(\Omega, \mathfrak{I}, \mathbb{P})$ be a complete probability space. A standard fractional Brownian motion $\left\{\beta^{\mathrm{H}}(t), t \in \mathbb{R}\right\}$ with Hurst parameter $\mathrm{H} \in(0,1)$ is a zero mean Gaussian process with the covariance function

$$
R_{\mathrm{H}(t, s)}=\mathbf{E}\left[\beta^{\mathrm{H}}(t) \beta^{\mathrm{H}}(s)\right]=\frac{1}{2}\left(|t|^{2 \mathrm{H}}+|s|^{2 \mathrm{H}}-|t-s|^{2 \mathrm{H}}\right), \quad t, s \in \mathbb{R} .
$$

Let $\mathrm{H}$ and $\mathrm{K}$ be two real separable Hilbert spaces and let $\mathscr{L}(\mathrm{K}, \mathrm{H})$ be the space of bounded linear operator from $\mathrm{K}$ to $\mathrm{H}$. Let $Q \in \mathscr{L}(\mathrm{H}, \mathrm{K})$ be an operator defined by $Q e_{n}=\lambda_{n} e_{n}$ with finite trace $\operatorname{tr} Q=\sum_{n=1}^{\infty} \lambda_{n}<\infty$. where $\lambda_{n} \geq 0(n=1,2, \ldots)$ are non-negative real numbers and $\left\{e_{n}\right\}$ $(n=1,2, \ldots)$ is a complete orthonormal basis in $\mathrm{K}$. We define the infinite dimensional fractional Brownian motion on $\mathrm{K}$ with covariance $Q$ as $\mathrm{B}^{\mathrm{H}}(t)=\mathrm{B}_{Q}^{\mathrm{H}}(t)=\sum_{n=1}^{\infty} \sqrt{\lambda_{n}} e_{n} \beta_{n}^{\mathrm{H}}(t)$. where $\beta_{n}^{\mathrm{H}}$ are real, independent fractional Brownian motion's. This process is Gaussian, it starts from 0 , has zero mean and covariance

$$
\mathbf{E}\left\langle\mathrm{B}^{\mathrm{H}}(t), x\right\rangle\left\langle\mathrm{B}^{\mathrm{H}}(s), y\right\rangle=R(s, t)\langle Q(x), y\rangle \text { for } x, y \in \mathrm{Y} \text { and } t, s \in[0, T]
$$

Now, define the Weiner integrals with respect to the $Q$-fractional Brownian motion, we introduce the space $\mathscr{L}_{2}^{0}=\mathscr{L}_{2}^{0}(\mathrm{~K}, \mathrm{H})$ of all $Q$-Hilbert-Schmidt operators $\zeta: \mathrm{K} \rightarrow \mathrm{H}$. We recall that $\zeta \in \mathscr{L}(\mathrm{K}, \mathrm{H})$ is called a $Q$-Hilbert-Schmidt operator, if

$$
\|\zeta\|_{\mathscr{L}_{2}^{0}}^{2}=\sum_{n=1}^{\infty}\left\|\sqrt{\lambda_{n}} \zeta e_{n}\right\|^{2}<\infty
$$

and that the space $\mathscr{L}_{2}^{0}$ equipped with the inner product $\left\langle\varphi, \zeta>_{\mathscr{L}_{2}^{0}}=\sum_{n=1}^{\infty}<\varphi e_{n}, \zeta e_{n}>\right.$ is a separable Hilbert space. Let $\phi(s): s \in[0, T]$ be a function with values in $\mathscr{L}_{2}^{0}(\mathrm{~K}, \mathrm{H})$ such that $\sum_{n=1}^{\infty}\left\|K^{*} \phi Q^{1 / 2} e_{n}\right\|_{\mathscr{L}_{2}^{0}}^{2}<\infty$. The Weiner integral of $\phi$ with respect to $\mathrm{B}^{\mathrm{H}}$ is defined by

$$
\int_{0}^{t} \phi(s) d \mathrm{~B}^{\mathrm{H}}=\sum_{n=1}^{\infty} \int_{0}^{t} \sqrt{\lambda_{n}} \phi(s) e_{n} d \beta_{n}^{\mathrm{H}}(s) .
$$

Lemma 2.1. If $\zeta:[0, T] \rightarrow \mathscr{L}_{2}^{0}(\mathrm{~K}, \mathrm{H})$ satisfies $\int_{0}^{t}\|\zeta(s)\|_{\mathscr{L}_{2}^{0}}^{2} d s<\infty$, then $(2.1)$ is well defined as an $\mathrm{H}$-valued random variable and

$$
\mathbf{E}\left\|\int_{0}^{t} \zeta(s) d \mathrm{~B}^{\mathrm{H}}(s)\right\|^{2} \leq 2 \mathrm{H} t^{2 \mathrm{H}-1} \int_{0}^{t}\|\zeta\|_{\mathscr{L}_{2}^{0}}^{2} d s .
$$

Let $r>0$ and $\mathscr{C}:=\mathscr{C}([-r, 0] ; \mathrm{H})$ denotes the family of all continuous functions from $[-r, 0]$ to $\mathrm{H}$. The space $\mathscr{C}$ is assumed to be equipped with the norm

$$
\|\zeta\|_{\mathscr{C}}:=\sup _{-r<t<0}\|\zeta(t)\|_{\mathrm{H}}, \quad \zeta(t) \in \mathscr{C} .
$$

We also assume that $\mathscr{C}_{\mathfrak{I}_{0}}^{b}([-r, 0] ; \mathrm{H})$ denotes the family of all almost surely bounded, $\mathfrak{I}_{0}$-measurable, $\mathscr{C}([-r, 0] ; \mathrm{H})$-value random variables. For all $t \geq 0, x_{t}=\{x(t+\theta):-r \leq \theta \leq 0\}$ is regarded as $\mathscr{C}([-r, 0] ; \mathrm{H})$-valued stochastic process. Further, let $\mathscr{P} \mathscr{C}\left(J, \mathscr{L}^{2}(\Omega, \mathrm{H})\right)=\{x(t)$ is continuous everywhere except for some $t_{k}$ at which $x\left(t_{k}^{-}\right)$and $x\left(t_{k}^{+}\right)$exist and $\left.x\left(t_{k}^{-}\right)=x\left(t_{k}\right), k=1,2, \ldots, m\right\}$ be the Banach space of piece-wise continuous function from $J$ into $\mathscr{L}^{2}(\Omega, \mathrm{H})$ with the norm

$$
\|x\|_{\mathscr{P} \mathscr{C}}=\sup _{t \in J}|x(t)|<\infty .
$$


Let $\mathscr{P} \mathscr{C}=\mathscr{P} \mathscr{C}\left(J, \mathscr{L}^{2}\right)$ be the closed subspace of $\mathscr{P} \mathscr{C}\left(J, \mathscr{L}^{2}(\Omega, \mathrm{H})\right)$ consisting of measurable and $\mathfrak{I}_{t}$-adapted H-valued process $x(\cdot) \in$ $\mathscr{P} \mathscr{C}\left(J, \mathscr{L}^{2}(\Omega, \mathrm{H})\right)$ endowed with the norm

$$
\|x\|_{\mathscr{P} \mathscr{C}}^{2}=\mathbf{E} \sup _{t \in J}\|x(t)\|_{\mathrm{H}}^{2} .
$$

In what follows, we assume that $\{A(t), t \geq 0\}$ is a family of closed densely defined linear unbounded operators on $\mathrm{H}$ and with domain $\mathscr{D}=\mathscr{D}(A(t))$ independent of $t$.

Definition 2.2. A family of bounded linear operators $\{\mathscr{U}(t, s)\}_{(t, s) \in \Delta}: \mathscr{U}(t, s): \mathrm{H} \rightarrow \mathrm{H}$ for $(t, s) \in \Delta:=\{(t, s) \in J \times J: 0 \leq s \leq t \leq T\}$ is called an evolution system if the following properties are satisfied

1. $\mathscr{U}(t, t)=I$ where I is the identity operator in $\mathrm{H}$.

2. $\mathscr{U}(t, s) \mathscr{U}(s, r)=\mathscr{U}(t, r)$ for $0 \leq r \leq s \leq t \leq T$.

3. $\mathscr{U}(t, s) \in \mathscr{L}(\mathrm{H})$ the space of bounded linear operators on $\mathrm{H}$, where for every $(t, s) \in \Delta$ and for each $x \in \mathrm{H}$, the mapping $(t, s) \rightarrow \mathscr{U}(t, s) x$ is strongly continuous.

Remark 2.3. If $A(t), t \geq 0$ is a second order differential operator $A$, i.e. $A(t)=A$ for each $t \geq 0$. Then, A generates a $\mathscr{C}_{0}$-semigroup $\left\{e^{A t}, t \geq 0\right\}$.

More details on evolution systems and their properties could be found on the books of Pazy [5].

It is convenient to introduce the relevant operators and the basic controllability condition.

(i) The operator $\mathscr{L}_{0}^{T} \in \mathrm{L}\left(\mathscr{L}_{2}^{\mathfrak{I}}\right)\left(J, \mathrm{H}, \mathscr{L}_{2}\left(\Omega, \mathfrak{I}_{T}, \mathrm{H}\right)\right)$ is defined by

$$
\mathscr{L}_{0}^{T} u=\int_{0}^{T} \mathscr{U}(T, s) B u(s) d s
$$

where $\mathscr{L}_{2}^{\mathfrak{I}}(J, \mathrm{H})$ is the space of all $\mathfrak{I}_{t}$-adapted, H-valued measurable square integrable processes on $J \times \Omega$.

(ii) The linear controllability operator $\Pi_{0}^{T}$ which is associated with the operator $\mathscr{L}_{0}^{T}$ is defined by

$$
\Pi_{0}^{T}\{\cdot\}=\mathscr{L}_{0}^{T}\left(\mathscr{L}_{0}^{T}\right)^{*}\{\cdot\}=\int_{0}^{T} \mathscr{U}(T, t) B B^{*} \mathscr{U}^{*}(T, t) \mathbf{E}\left\{\cdot \mid \mathfrak{I}_{t}\right\} d t
$$

which belongs to $\mathrm{L}\left(\mathscr{L}_{2}^{\mathfrak{I}}\right)\left(J, \mathrm{H}, \mathscr{L}_{2}\left(\Omega, \mathfrak{I}_{T}, \mathrm{H}\right)\right)$ and the controllability operator $\Gamma_{s}^{T} \in \mathrm{L}(\mathrm{H}, \mathrm{H})$ is

$$
\Gamma_{s}^{T}=\int_{s}^{T} \mathscr{U}(T, t) B B^{*} \mathscr{U}^{*}(T, t) d t, \quad 0 \leq s \leq t .
$$

Lemma 2.4. For any $z \in \mathscr{L}_{2}\left(\Omega, \mathfrak{I}_{T}, \mathrm{H}\right)$, there exists $\bar{\phi} \in \mathscr{L}_{2}^{\mathfrak{I}}\left(J, \mathrm{~L}_{2}^{0}\right)$ such that

$$
z=\mathbf{E} z+\int_{0}^{T} \bar{\phi}(s) d \mathrm{~B}^{\mathrm{H}}(s) .
$$

Let $x(t ; \varphi, u)$ be the state value of the system (1.1)-(1.3) at terminal time $T$ corresponding to the control $u$ and the initial value $\varphi$. Introduce the set $\mathfrak{R}(T, \varphi)=\left\{x(T ; \varphi, u): u(\cdot) \in \mathscr{L}_{2}^{\mathfrak{I}}(J, \mathrm{U})\right\}$ is called the reachable set of the system (1.1)-(1.3).

Definition 2.5. The system (1.1)-(1.3) is said to be approximately controllable on the interval $J$ if $\overline{\Re(T, x)}=\mathscr{L}_{2}\left(\Omega, \mathfrak{I}_{T}, \mathrm{H}\right)$

Definition 2.6. An $\mathfrak{I}_{t}$-adapted stochastic process $x: J \rightarrow \mathrm{X}$ is called a mild solution of (1.1)-(1.3) if for each $u \in \mathscr{L}_{2}^{\mathfrak{I}}(J, \mathrm{U})$ and for $t \in J$, $\mathbf{P}\left\{\omega: \int_{J}\|x(s)\|_{\mathrm{X}}^{2} d s<+\infty\right\}=1$ it satisfies the integral equation

$$
\begin{aligned}
x(t) & =\mathscr{U}(t, 0)[\varphi(0)-g(0, \varphi)]+g(t, x(t-r))+\int_{0}^{t} \mathscr{U}(t, s) f(s, x(s), x(s-r)) d s \\
& +\int_{0}^{t} \mathscr{U}(t, s) B u(s) d s+\int_{0}^{t} \mathscr{U}(t, s) \sigma(s) d \mathrm{~B}^{\mathrm{H}}(s)+\sum_{0<t_{k}<t} \mathscr{U}\left(t, t_{k}\right) I_{k}\left(x\left(t_{k}^{-}\right)\right), \quad t \in[0, T] .
\end{aligned}
$$

We improve the following hypotheses to prove our results:

(H1) $\mathscr{U}(t, s)$ is a compact operator for $t-s>0$ and there exists a constant $k \geq 1$ such that

$$
\|\mathscr{U}(t, s)\|_{\mathscr{L}(\mathrm{H})} \leq k, \text { for }(t, s) \in \Delta .
$$

(H2) There exists a positive constant $k_{0}$ such that $t \in J, x, y \in \mathrm{H}$

$$
\|g(t, x)-g(t, y)\|_{\mathrm{H}}^{2} \leq k_{0}\|x-y\|_{\mathrm{H}}^{2} .
$$

(H3) There exists a positive constant $k_{1}$ such that for all $x_{1}, x_{2}, y_{1}, y_{2} \in \mathrm{H}$

$$
\|f(t, x)-f(t, y)\|_{\mathrm{H}}^{2} \leq k_{1}\left(\left\|x_{1}-x_{2}\right\|_{\mathrm{H}}^{2}+\left\|y_{1}-y_{2}\right\|_{\mathrm{H}}^{2}\right) .
$$

(H4) There exists some positive constants $Q_{k}, k=1,2, \ldots, m$ such that for $x, y \in \mathrm{H}$

$$
\left\|I_{k}(x)-I_{k}(y)\right\|_{\mathrm{H}}^{2} \leq Q_{i}\|x-y\|_{\mathrm{H}}^{2} .
$$


(H5) For all $t \in J$, there exists a positive constant $l$ such that

$$
\|g(t, 0)\|_{\mathrm{H}}^{2} \vee\|f(t, 0)\|_{\mathrm{H}}^{2} \vee\left\|I_{k}(0)\right\|_{\mathrm{H}}^{2} \leq l .
$$

(H6) The function $\sigma:[0, T] \rightarrow \mathscr{L}_{2}^{0}(\mathrm{~K}, \mathrm{H})$ satisfies

$$
\int_{0}^{t}\|\sigma(s)\|_{\mathscr{L}_{2}^{0}}^{2} d s<\infty, \text { for } T>0 .
$$

(H7) For $0 \leq t \leq T$, the operator $a R\left(a, \Gamma_{t}^{T}\right):=a\left(a I+\Gamma_{t}^{T}\right)^{-1} \rightarrow 0$ as $a \rightarrow 0^{+}$in the strong operator topology.

(H8) The functions $f, g$ are uniformly bounded.

Remark 2.7. The assumption (H7) is equivalent to the linear system of (1.1)-(1.3) is approximately controllable.

\section{Controllability Result}

Let $a>0$ and $z \in \mathscr{L}_{2}\left(\Omega, \mathfrak{I}_{T}, \mathrm{H}\right)$. Define the control function

$$
\begin{aligned}
u_{a}(t, x) & =B^{*} \mathscr{U}^{*}(T, t)\left[R\left(a, \Pi_{0}^{T}\right)(\mathbf{E} z-\mathscr{U}(T, 0)[\varphi(0)-g(0, \varphi)]-g(t, x(t-r))\right. \\
& \left.\left.-\sum_{0<t_{k}<T} \mathscr{U}\left(T, t_{k}\right) I_{k}\left(x\left(t_{k}^{-}\right)\right)\right)+\int_{0}^{T} R\left(a, \Pi_{s}^{T}\right) z(s) d \mathrm{~B}^{\mathrm{H}}(s)-\int_{0}^{T} R\left(a, \Pi_{s}^{T}\right) \mathscr{U}(T, s) f(s, x(s), x(s-r)) d s\right]
\end{aligned}
$$

Theorem 3.1. Suppose that (H1)-(H8) hold. Then the operator $\Theta$ has a fixed point in $\mathscr{P} \mathscr{C}$ provided that

$$
4\left(\frac{a^{2}+3 T^{2} k^{4} k_{B}^{2}}{a^{2}}\right)\left[k_{0}+2 T^{2} k^{2} k_{1}+m k^{2} \sum_{k=1}^{m} Q_{k}\right]\|x-y\|_{\mathscr{P} \mathscr{C}}^{2}<1 .
$$

Proof. Consider the operator $\Theta: \mathscr{P} \mathscr{C}\left(J, \mathscr{L}^{2}(\Omega ; \mathrm{H})\right) \rightarrow \mathscr{P} \mathscr{C}\left(J, \mathscr{L}^{2}(\Omega ; \mathrm{H})\right)$ defined by

$$
\begin{aligned}
(\Theta x)(t) & =\mathscr{U}(t, 0)[\varphi(0)-g(0, \varphi)]+g(t, x(t-r))+\int_{0}^{t} \mathscr{U}(t, s) f(s, x(s), x(t-r)) d s \\
& +\int_{0}^{t} \mathscr{U}(t, s) B u_{a}(s, x) d s+\int_{0}^{t} \mathscr{U}(t, s) \sigma(s) d \mathrm{~B}^{\mathrm{H}}(s)+\sum_{0<t_{k}<t} \mathscr{U}\left(t, t_{k}\right) I_{k}\left(x\left(t_{k}^{-}\right)\right) .
\end{aligned}
$$

In what follows, we shall show that system (1.1)-(1.3) is approximately controllable if for all $a>0$ there exists a fixed point of the operator $\Theta$. By our assumptions, Holder's inequality and the Doob martingale inequality, we obtain

$$
\begin{aligned}
\mathbf{E}\left\|u_{a}(t, x)\right\|^{2} & \leq \frac{7}{a^{2}} k^{2} k_{B}^{2}\left[\|\mathbf{E} z\|^{2}+2 k^{2}\left[1+k_{0}\right] \mathbf{E}\|\varphi\|_{\mathscr{P} \mathscr{C}}^{2}+2\left[M+k_{0}\right]\left(\mathbf{E}\|\varphi\|_{\mathscr{P} \mathscr{C}}^{2}+\|x\|_{\mathscr{P} \mathscr{C}}^{2}\right)\right. \\
& +2 m k^{2} \sum_{k=1}^{m} Q_{k}\left(M+\|x\|_{\mathscr{P} \mathscr{C}}^{2}\right)+k^{2} 2 \mathrm{H} t^{2 \mathrm{H}-1} \int_{0}^{t}\|\sigma(s)\|_{\mathscr{L}_{2}^{0}}^{2} d s \\
& \left.+2 k^{2} T\left[M T+k_{1}\left(r \mathbf{E}\|\varphi\|_{\mathscr{P} \mathscr{C}}^{2}+2 T\|x\|_{\mathscr{P} \mathscr{C}}^{2}\right)\right]\right]
\end{aligned}
$$

Step 1: We claim that $\Theta(\mathscr{P} \mathscr{C}) \subset \mathscr{P} \mathscr{C}$. Then we have

$$
\begin{aligned}
& \|(\Theta x)(t)\|_{\mathscr{P} \mathscr{C}}^{2} \leq 6\left[k^{2}\left(\|\varphi(0)-g(0, \varphi)\|^{2}\right)+\mathbf{E}\|g(t, x(t-r))-g(t, 0)+g(t, 0)\|^{2}\right. \\
& +k^{2} T \mathbf{E} \int_{0}^{t}\|f(s, x(s), x(s-r))-f(t, 0,0)+f(t, 0,0)\|^{2} d s+k^{2} k_{B}^{2} T^{2} \mathbf{E}\left\|u_{a}(s, x)\right\|^{2} \\
& \left.+2 k^{2} \mathrm{H} t^{2 \mathrm{H}-1} \int_{0}^{t}\|\sigma(s)\|_{\mathscr{L}_{2}^{0}}^{2} d s+k^{2} \mathbf{E} \sum_{0<t_{k}<t}\left\|I_{x}\left(x\left(t_{k}^{-}\right)\right)-I_{k}(0)+I_{k}(0)\right\|^{2}\right] \\
& \leq 6\left[\frac{7}{a^{2}} T^{2} k^{4} k_{B}^{4}\left(\|\mathbf{E} z\|^{2}\right)+\left[1+\frac{7}{a^{2}} T^{2} k^{4} k_{B}^{4}\right]\left(2 k^{2}\left(1+k_{0}\right) \mathbf{E}\|\varphi\|_{\mathscr{P} \mathscr{C}}^{2}\right.\right. \\
& +2\left[M+k_{0}\left(\mathbf{E}\|\varphi\|_{\mathscr{P} \mathscr{C}}^{2}+\|x\|_{\mathscr{P} \mathscr{C}}^{2}\right)\right]+2 m k^{2} \sum_{k=1}^{m} Q_{k}\left(M+\|x\|_{\mathscr{P} \mathscr{C}}^{2}\right) \\
& \left.\left.+2 k^{2} T\left[M T+k_{1}\left(\mathbf{E}\|\varphi\|_{\mathscr{P} \mathscr{C}}^{2}+\|x\|_{\mathscr{P} \mathscr{C}}^{2}\right)\right]+2 k^{2} \mathrm{H} t^{2 \mathrm{H}-1} \int_{0}^{t}\|\sigma(s)\|_{\mathscr{L}_{2}^{0}}^{2} d s\right)\right]<\infty .
\end{aligned}
$$

Hence, $\Theta(\mathscr{P} \mathscr{C}) \subset \mathscr{P} \mathscr{C}$.

Now, we are going to use the Banach fixed point theorem to prove that $\Theta$ has a unique fixed point in $\mathscr{P} \mathscr{C}$.

Step 2: We claim that $\Theta$ is a contraction on $\mathscr{P} \mathscr{C}$.

For any $x, y \in \mathscr{P} \mathscr{C}, t \in J$ then we have

$$
\begin{aligned}
\|(\Theta x)(t)-(\Theta y)(t)\|_{\mathscr{P} \mathscr{C}}^{2} & \leq 4\left[k_{0}+2 T^{2} k^{2} k_{1}+m k^{2}\right]\|x-y\|_{\mathscr{P} \mathscr{C}}^{2}+4 T^{2} k^{2} k_{B}^{2} \mathbf{E}\left\|u_{a}(t, x)-u_{a}(t, y)\right\|_{\mathrm{H}}^{2} \\
& \leq 4\left(\frac{a^{2}+3 T^{2} k^{4} k_{B}^{2}}{a^{2}}\right)\left[k_{0}+2 T^{2} k^{2} k_{1}+m k^{2} \sum_{k=1}^{m} Q_{k}\right]\|x-y\|_{\mathscr{P} \mathscr{C}}^{2} .
\end{aligned}
$$


By (3.1), we conclude that $\Theta$ is a contraction mapping on $\mathscr{P} \mathscr{C}$. Thus by the Banach fixed point theorem, has a unique fixed point $x(\cdot) \in \mathscr{P} \mathscr{C}$. Hence the proof.

Theorem 3.2. Assume the condition in Theorem 3.1 and $\mathbf{( H 8 )}$ are satisfied, then system (1.1)-(1.3) is approximately controllable on $[0, T]$.

Proof. By Theorem 3.1, $\Theta$ has a unique fixed point $x_{a}^{*}$ in $\mathscr{P} \mathscr{C}$. By using the stochastic Fubini theorem, it can easily be seen that

$$
\begin{aligned}
x_{a}^{*}(T) & =z-a R\left(a, \Pi_{0}^{T}\right)\left[\mathbf{E} z-\mathscr{U}(T, 0)[\varphi(0)-g(0, \varphi)]-g\left(t, x^{*}(t-r)\right)+\int_{0}^{T} \bar{\phi}(s) d \mathrm{~B}^{\mathrm{H}}(s)\right] \\
& +\int_{0}^{T} a R\left(a, \Pi_{s}^{T}\right) \mathscr{U}(T, s) f\left(s, x^{*}, x^{*}(s-r)\right) d s \\
& +\int_{0}^{T} a R\left(a, \Pi_{s}^{T}\right) \mathscr{U}(T, s) \sigma(s) d \mathrm{~B}^{\mathrm{H}}(s)+\sum_{0<t_{k}<t} \mathscr{U}\left(T, t_{k}\right) I_{k}\left(x^{*}\left(t_{k}^{-}\right)\right) .
\end{aligned}
$$

It follows from the assumption (H8) that there exists $\overline{\mathscr{K}}$ such that

$$
\left\|f\left(s, x^{*}(s), x^{*}(s-r)\right)\right\|_{\mathrm{H}}^{2}+\left\|g\left(s, x^{*}(t-r)\right)\right\|_{\mathscr{L}_{2}^{0}}^{2} .
$$

Then there is a subsequence still denoted by $\left\{f\left(s, x^{*}(s), x^{*}(s-r)\right), g\left(s, x^{*}(s-r)\right)\right\}$ which converges weakly to say, $\{f(s), g(s)\}$ in $\mathrm{H} \times \mathscr{L}_{2}^{0}$. On the other hand, by (H7), for all $o \leq t \leq T, a R\left(a, \Pi_{s}^{T}\right) \longrightarrow 0$ as $a \rightarrow 0^{+}$strongly and moreover $\left\|a R\left(a, \Pi_{s}^{T}\right)\right\| \leq 1$. Therefore, by the Lebesgue dominated convergence theorem and the compactness of $\mathscr{U}(\cdot, \cdot)$ it follows that

$$
\begin{aligned}
\mathbf{E}\left\|x_{a}^{*}(T)-z\right\|^{2} & \leq 5 \mathbf{E} \| a R\left(a, \Pi_{0}^{T}\right)\left(\mathbf{E} z-a R\left(a, \Pi_{0}^{T}\right)\right)\left[\mathbf{E} z-\mathscr{U}(T, 0)[\varphi(0)-g(0, \varphi)]-g\left(t, x^{*}(t-r)\right)\right. \\
& \left.+\int_{0}^{T} \bar{\phi}(s) d \mathrm{~B}^{\mathrm{H}}(s)\right] \|^{2}+5 \mathbf{E}\left(\int_{0}^{T}\left\|a R\left(a, \Pi_{s}^{T}\right) \mathscr{U}(T, s)\left[f\left(s, x^{*}(s), x^{*}(s-r)\right)-f(s)\right]\right\| d s\right)^{2} \\
& +5 \mathbf{E}\left(\int_{0}^{T}\left\|a R\left(a, \Pi_{s}^{T}\right) \mathscr{U}(T, s) f(s)\right\| d s\right)^{2}+10 \mathrm{H} T^{2 \mathrm{H}-1} \int_{0}^{T}\left\|a R\left(a, \Pi_{s}^{T}\right) \mathscr{U}(T, s) \sigma(s)\right\|_{\mathscr{L}_{2}^{0}}^{2} d s \\
& +5 \mathbf{E}\left\|\sum_{0<t_{k}<T} a R\left(a, \Pi_{s}^{T}\right) \mathscr{U}\left(T, t_{k}\right) I_{k}\left(x^{*}\left(t_{k}^{-}\right)\right)\right\|^{2} \longrightarrow 0 \text { as } a \rightarrow 0^{+} .
\end{aligned}
$$

Thus, $x_{a}^{*}(T) \rightarrow z$ holds in $\mathrm{H}$ and consequently we obtain the approximate controllability of system (1.1)-(1.3).

\section{An Example}

We consider the following stochastic classical heat equation with memory of the form:

$$
\begin{aligned}
d[u(t, \zeta)-\bar{g}(t, u(t-r), \zeta)] & =\left[\frac{\partial^{2}}{\partial \zeta^{2}} u(t, \zeta)+a(t, \zeta) u(t, \zeta)-\bar{g}(t, u(t-r), \zeta)\right] d t \\
+ & f(t, u(t, \zeta), u(t-r), \zeta) d t+\mu(t, \zeta) d t+\sigma(t) d \mathrm{~B}^{\mathrm{H}}(t), \zeta \in[0, \pi], t \neq t_{k}, J=[0, T] \\
u\left(t_{k}^{+}, \zeta\right)-u\left(t_{k}^{-}, \zeta\right) & =I_{k}\left(u\left(t_{k}^{-}, \zeta\right)\right), \quad k \in\{1,2, \ldots, m\} \\
u(t, 0) & =u(t, \pi)=0, t \in[0, T], \\
u(\theta, \cdot) & =\varphi(\theta, \cdot) \in \mathrm{H}=\mathscr{L}^{2}[0, \pi], \quad \varphi(\cdot, \zeta) \in \mathscr{C}([-r, 0] ; \mathbb{R}), \quad \theta \in[0, \pi] .
\end{aligned}
$$

where $\mathrm{B}^{\mathrm{H}}$ is a fractional Brownian motion and let $\mathrm{H}=\mathrm{K}=\mathrm{U}=\mathscr{L}^{2}([0, \pi])$. Define $A: \mathrm{H} \rightarrow \mathrm{H}$ by $A x=x^{\prime \prime}$ with domain $\mathscr{D}(A)=\{x \in \mathrm{H}$ : $x, x^{\prime}$ are absolutely continuous $\left.x^{\prime \prime} \in \mathrm{H}, x(0)=x(\pi)=0\right\}$. The spectrum of $A$ consists of the eigenvalues $-n^{2}$ for $n \in \mathbb{N}$, with associated eigenvectors $e_{n}=\sqrt{\frac{2}{\pi}} \sin n x, n=1,2,3, .$. It is wellknown that $A$ is the infinitesimal generator of a strongly continuous semigroup $\{S(t)\}$, $t \geq 0$ on $\mathrm{H}$ is given by

$$
S(t) x=\sum_{n=1}^{\infty} e^{-n^{2}}<x, e_{n}>e_{n}, \quad x \in \mathrm{H} .
$$

Now, define the fractional Brownian motion in $\mathrm{K}$ by

$$
\mathrm{B}^{\mathrm{H}}(t)=\sum_{n=1}^{\infty} \sqrt{\lambda_{n}} \beta^{\mathrm{H}}(t) e_{n},
$$

where $\mathrm{H} \in(1 / 2,1)$ and $\left\{\beta_{n}^{\mathrm{H}}\right\}_{n \in \mathbb{N}}$ is a sequence of one dimensional fractional Brownian motions mutually independent. On the domain $\mathscr{D}(A)$, we define the operators $A(t): \mathscr{D}(A) \subset \mathrm{H} \rightarrow \mathrm{H}$ by $A(t) z(\zeta)=A z(\zeta)+a(t, \zeta) z(\zeta)$. Let $a(\cdot, \cdot)$ be continuous and $a(t, \zeta) \leq-\lambda, \lambda>0$ for all $t \in J, \zeta \in[0, \pi]$, it follows that the system

$$
\left\{\begin{array}{l}
d u(t)=A(t) u(t) d t, \quad t \geq s, \\
u(s)=z \in \mathrm{H} .
\end{array}\right.
$$


has an associated evolution family $\{\mathscr{U}(t, s)\}_{t \geq s}$ as $\mathscr{U}(t, s) z(\zeta)=\left(S(t-s) e^{\int_{s}^{t} a(p, \zeta) d t} z\right)(\zeta)$

From the above expression, it follows that $\mathscr{U}(t, s)$ is a compact operator and every $t, s \in J$ with $t>s$

$$
\|\mathscr{U}(t, s)\| \leq e^{-(1+\lambda)(t-s)} .
$$

Thus, (H1) is true.

Now we define the linear continuous mapping $B: \mathrm{U} \rightarrow \mathrm{H}$ by $u(t)=\mu(t, \zeta)$ where $\mu(t, \zeta): J \times[0, \pi] \rightarrow[0, \pi]$ is continuous.

Define $x(t)(\cdot)=u(t, \cdot), f(t, u, u)(\cdot)=\bar{f}(t, u, u)(\cdot), g(t, u)(\cdot)=\bar{g}(t, u)(\cdot)$. Then, under the above conditions, we can represent the stochastic control system (4.1) in the abstract from (1.1)-(1.3). Thus we can conclude that the stochastic control system (4.1) is approximately controllable on $[0, T]$ provided that all the conditions of Theorem 3.2 are satisfied.

\section{References}

[1] H. M. Ahmed, Approximate controllability of impulsive neutral stochastic differential equations with fractional Brownian motion in a Hilbert space, Adv. Difference Equ., 113 (2014), 1-11.

[2] H. M. Ahmed, Controllability of impulsive neutral stochastic differential equations with fractional Brownian motion, IMA J. Math. Control Inform, 32(4) (2015), 781-794

[3] A. Boudaoui, E. Lakhel, Controllability of stochastic impulsive neutral functional differential equations driven by fractional Brownian motion with infinite delay, Differ. Equ. Dyn. Syst., 26 (2018), 247-263.

[4] G. Da Prato, J. Zabezyk, Stochastic Equations in Infinite Dimensions, Cambridge: University Press, Cambridge, UK, 44 (1992).

[5] A. Pazy, Semigroups of linear operators and applications to partial differential equations, Appl. Math. Sci, Springer-Verlag, New York, 44 (1983).

[6] V. Lakshmikantham, D. D. Bainor and P. S. Simeonnov, Theory of impulsive differential equations, World Scientific, (1989).

[7] N. I. Mahmudov, S. Zorlu, Controllability of nonlinear stochastic systems, Int. J. Control, 76(2) (2003), 95-104.

[8] N. I. Mahmudov, On controllability of linear stochastic system in Hilbert space, J. Math. Anal. Appl., 259 (2001), 64-82.

[9] A. N. Kolmogorov, Wienerschc Spiralen and einige andere interessante Kurven in Hilbertsehen Raum, C.R.(Doklady)Acad.URSS(N.S), 26 (1940), $115-118$.

[10] B. B. Mandelbrot, J. W. Van Ness, Fractional Brownian motions, fractional noise and applications, SIAM Rev, 10 (1968), $422-437$.

[11] E. Lakhel, Controllability of neutral stochastic functional differential equations driven by fractional Brownian motion with infinite delay, Nonlinear Dyn. Syst. Theory, 17(3) (2017), 291-302.

[12] E. Lakhel, Controllability of neutral stochastic functional integrodifferential equations driven by fractional Brownian motion, Stoch. Anal. Appl., 34(3) (2016), 427-440.

[13] A. Anguraj, K. Ramkumar, Approximate controllability of semilinear stochastic integrodifferential system with nonlocal conditions, Fractal Fract, 2(4) (2018), 29

[14] R. Sakthivel, R. Ganesh, Y. Ren and S. M. Anthoni, Approximate controllability of nonlinear fractional dynamical systems, Commun. Nonlinear Sci. Numer. Simul., 18 (2013), 3498-3508.

[15] M. Chen, Approximate controllability of stochastic equations in a Hilbert space with fractional Brownian motion, Stoch. Dyn., 15 (2015), 1-16. 